\title{
HYPERSPECTRAL IMAGE MIXED NOISE REDUCTION BASED ON IMPROVED K-SVD ALGORITHM
}

\author{
S. Shajun Nisha ${ }^{1}$, P. Thazneem Fazila ${ }^{2}$, S. Kother Mohideen ${ }^{3}$ \\ ${ }^{1}$ Professor and Head, Department of Computer Science (PG), Sadakathullah Appa College, Tamil Nadu, India \\ ${ }^{2}$ Student, Computer Science and Engineering, National College of Engineering, Tamil Nadu, India \\ ${ }^{3}$ Professor, Computer Science and Engineering, National College of Engineering, Tamil Nadu, India
}

\begin{abstract}
We propose an algorithm for mixed noise reduction in Hyperspectral Imagery (HSI). The hyperspectral data cube is considered as a three order tensor. These tensors give a clear view about both spatial and spectral modes. The HSI provides ample spectral information to identify and distinguish spectrally unique materials, thus they are spectrally over determined. Tensor representation is three ordered thus can simultaneously deal with the two spatial dimensions and one spectral dimension of HSI to achieve a satisfying noise reduction performance. The image analysis application like Classification, unmixing, subpixel mapping and target detection are performed in a very accurate manner due to the development of hyperspectral remote sensing technology as it provides large amount of spatial and spectral information. This entire denoising process is based on the K-SVD denoising algorithm. This method of denoising can efficiently remove a variety of mixed or single noise by applying sparse regularization of small image patches. It also maintains the image texture in a clear manner. The learned dictionary used clearly helps in removing the noise. Our work involved in minimizing model to remove mixed noise such as Impulse noise, Gaussian-Gaussian mixture and Gaussian-Impulse noise from the HSI data. The weighted rank-one approximation problem is solved using a new iterative scheme and the low rank approximation can be obtained by singular value decomposition(SVD).A new weighting data fidelity function which is much easier to optimize is used which has the same minimizer as the original likelihood function. The weighting function in the model can be determined by the algorithm itself, and it plays a role of noise detection in terms of the different estimated noise parameters.
\end{abstract}

Keywords: Key Hyperspectral image, K-SVD algorithm, low rank approximation, Gaussian noise, Impulse noise, mixed noise

\section{INTRODUCTION}

The 'Hyper' means 'over' and refers to a large number of measured wavelength bands. The acquired HSI images are mostly corrupted by radiometric noise such as calibration error, atmospheric scattering, sensor noise, photon noise and absorption. There are two category of noise which affects HSI images they are the random noise and fixed-pattern noise. Fixed pattern noise is mostly due to calibration can be mitigated with suitable methods. In contrast, random noise due to its stochastic nature cannot be removed entirely. The random noise in HSI is the additive model, which is assumed to be white, Gaussian and independent-fromsignal.

Some of the traditional denoising algorithms are channel by channel, singular value decomposition (SVD), Wiener and wavelet filters. However these do not deal with the spatial and spectral information simultaneously and may lead to loss of the inter-dimensional information. In recent years, some algorithm combines the spatial and spectral information for HSI noise reduction. The algorithm like hybrid spatial spectral derivative domain wavelet shrinkage noise reduction (HSSNR) approach and spectral- spatial adaptive total variation model considers both spatial and spectral information in hyperspectral image for denoising.
The spatial and spectral information are completely preserved in multilinear algebra since the HSI data cube can be considered as a three order tensor. The multidimensional filtering based on tucker tensor decomposition is an example of such approach. One of the Tucker based noise reduction is the multidimensional Wiener filtering (MWF) algorithm [13] which achieves simultaneous improvement in the image quality and classification accuracy. However this application may lead to information compression and loss of spatial details. In the rank-1 tensor decomposition (R1TD) algorithm [24], the input data cube is considered as three order tensor. Subsequently, it sorts the eigen values generated by tensor decomposition and then extract the signal-dominant component from the observed HSI data cube. These signal-dominant components are extracted from the data cube by sorting the weights of the rank-1tensor, and then they are reconstructed to produce the noise free image.

In this work, we propose a general framework to adaptively detect and remove noise of different type, including Gaussian noise, impulse noise and more importantly, their mixture in the HSI data. The HSI data is considered as a three order tensor which treats both spatial and spectral modes of the given image. The image undergoes tensor decomposition; later the modified K-SVD algorithm is applied to the tensors. The regularized maximum likelihood 
estimation (MLE) is modified with new function along with additional variable, since the original likelihood functional related to mixed noise is not easy to be optimized compared with the functional for a single Gaussian noise. This new functional has the same global minimizer (or maximizer) as the original likelihood functional and is easier to be optimized. The weighting functions play the role of noise detectors by minimizing the new functional, we obtain some weighted norms models. We also integrate this with sparsity representation thus our model can well restore images and textures corrupted by mixed noise. A new iterative scheme is given to solve the weighted rank-one approximation problem arisen from the proposed model and the low rank approximation can be obtained by singular value decomposition (SVD). Our method integrates four steps as follows sparse coding followed by dictionary learning, image reconstruction, noise clustering (detection), and parameters estimation. Each step needs to perform a minimization problem. Then these optimized tensors are separated as noise free tensor and noisy tensor. The noise free tensors are then combined to reconstruct the noise free image. The reconstruction is same as reverse of the tensor decomposition.

The remainder of the paper is organized as follows. Section2 deals with brief review about tensor and its operation. Section 3 proposed method and section 4 provides the experimental results. Finally, section 5 concludes this study.

\section{BRIEF REVIEW ABOUT HSI AND TENSORS}

\subsection{Imaging Techniques}

RGB camera image is a type of multispectral image that uses the light intensity at three specific wavelengths: red, green, and blue, thus creating an image in the visible region. Thus depending on the number of spectral bands and wavelengths measured, an image is classified as a multispectral image when several wavelengths are measured. A hyperspectral image is complete wavelength region, i.e., the whole spectrum, is measured for each spatial point. For example, The Fig 1 compares the optical information obtained by black and white camera, RGB cameras, and hyperspectral cameras.

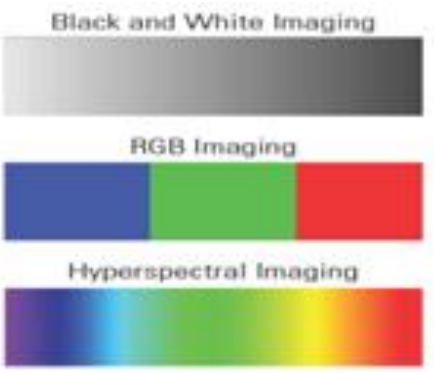

Fig-1: Difference in Imaging

\subsection{Imaging Spectrometer}

The instrument imaging spectrometers is used to produce the Hyperspectral images. These complex sensors have involved the convergence of two distinct but related technologies: spectroscopy and the remote imaging of Earth and planetary surfaces.

The study of light that is emitted by or reflected from materials and its variation in energy with wavelength is referred as Spectroscopy.

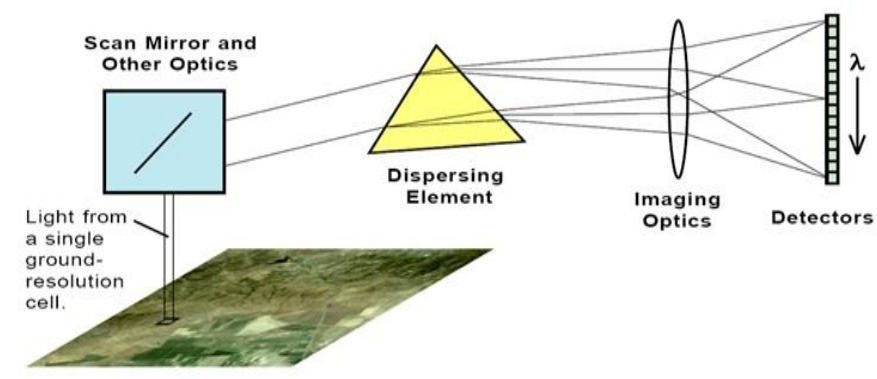

Fig-2: Imaging Spectrometer

In the field of optical remote sensing, spectroscopy deals with the spectrum of sunlight that is diffusely reflected (scattered) by materials at the Earth's surface. The groundbased or laboratory measurements of the light reflected from a test material are measured using the instrument like spectrometer (or spectrodiameter). The spectrometer consists of an optical dispersing element such as a grating or prism which splits this light into many narrow, adjacent wavelength bands. This energy in each band is measured by a separate detector. Spectrometers can make spectral measurements of bands as narrow as 0.01 micrometers over a wide wavelength range, typically at least 0.4 to 2.4 micrometers by using hundreds or even thousands of detectors.

\subsection{Tensors}

A tensor is defined as a multidimensional array and represented as $A \in R^{L_{1} \times L_{2} \times \ldots \ldots . . \times L_{N}}$. Tensor is the higher-order equivalent of the vector (one-order tensor) and a matrix (two-order tensor). In this study, the HSI data cube is a three-order tensor $A \in R^{L_{1} \times L_{2} \times L_{3}}$. The modes 1 and 2 belong to the spatial modes and mode 3 belongs to the spectral mode. Taking each vector to be in different mode the outer product of three vectors can be visualized as follows,

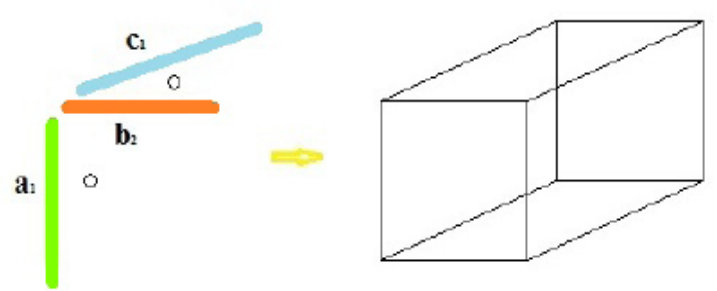

Fig-3: Outer product of three vectors forming a tensor

Mathematically, the outer product of three vectors $a ; b ; c$ is written as follows, 


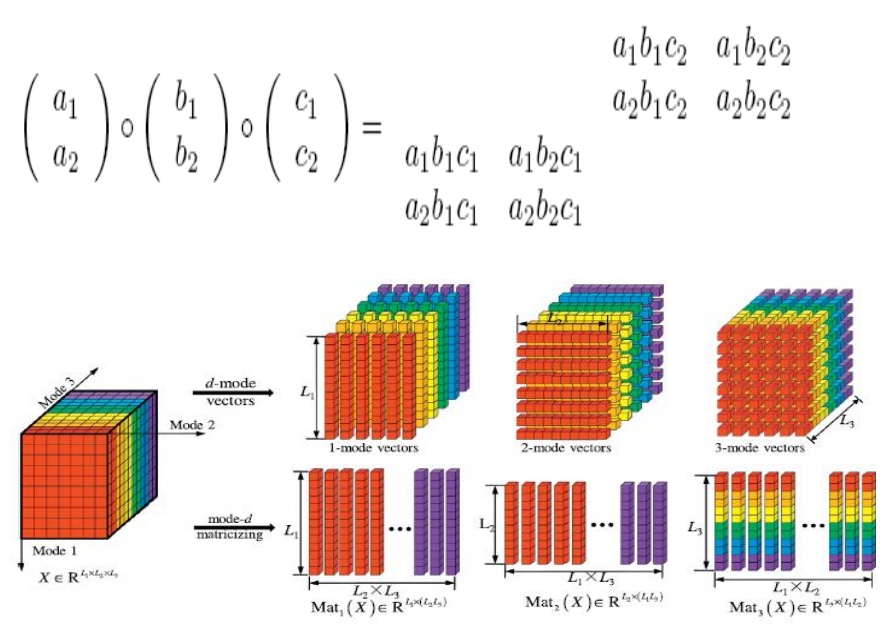

Fig-4: Tensor matricizationin three modes Tensor

matricization reorders tensor into a matrix. The n-mode matricization of $\mathrm{X}$ belongs to $\mathrm{RL} 1 \times \mathrm{L} 2 \times \ldots \times \mathrm{LN}$ is matnX belongs to $\mathrm{RLi} \times(\mathrm{L} 1 \mathrm{~L} 2 . . . \mathrm{Ln}-1 \mathrm{Ln}+1 \ldots \mathrm{LN})$, which is the ensemble of vectors in the n-mode obtained by keeping index Li fixed and varying the other indices. The tensor Matricization of three modes is shown in Fig 4.

\section{METHODOLOGY}

The given HSI is the input image to the system. This HSI image is read and displayed. Then the HSI image processed in the R1TD algorithm to provide the Rank-1 Tensor profiles. With these profiles, we perform the Alternative Least Square Algorithm to optimize the tensors. Then we sort the tensors of higher order and reconstruct the noise free image by combining signal dominant components.

\subsection{HSI Image Reader}

The Hyperspectral imaging (HSI) collects and process information from across the electromagnetic spectrum. The human eye sees visible light in three bands (red, blue, green). Spectral imaging divides the spectrum into many more bands. This technique of dividing images into bands can be extended before can be extended beyond the visibility. Hyperspectral sensors collect a set of images. Each image is a range of the electromagnetic spectrum known as a spectral band. Then these images are combined together to form a three dimensional hyperspectral data cube. This module is designed to read and visualize the HSI images.

The HSI data is considered as multiple images combined as a cube. Thus we have view each image in a well furnished manner. Each image ahs slice of images of different colors. This slice of image is not taken as a single color image for the calculation instead it is taken as whole cube called tensors.

Here $\mathrm{O}$ as the observed HSI data cube. This $\mathrm{O}$ consist both the noise free image $\mathrm{S}$ and the additive noise component $\mathrm{N}$. By extending the classic two-dimensional additive noise model, the tensorial formulation is,

$$
\mathrm{O}=\mathrm{S}+\mathrm{N}
$$

In this model, the noise is considered to be white, Gaussian and independent from signal.

\subsection{Tensor Decomposition}

New tensor decomposition is developed which jointly treats both the spatial and spectral modes. The R1TD algorithm is applied to the HSI data input which takes into account both the spatial and spectral information. The tensor decomposition is of the form CANDECOMP/PARAFAC decomposition (Canical decomposition and parallel factor decomposition).

According to the definitions of the rank 1 tensor and vector outer product, tensor $\mathrm{O} \in \mathrm{R}^{\mathrm{L}_{1} \times \mathrm{L}_{2} \times \mathrm{L}_{3}}$ can be represented with the rank-1 tensor decomposition model:

$\mathrm{O}=\sum_{\mathrm{r}=1}^{\mathrm{M}} \lambda_{\mathrm{r}} \mathrm{U}_{\mathrm{r}} \circ \mathrm{V}_{\mathrm{r}} \circ \mathrm{W}_{\mathrm{r}}$

Where $\mathrm{U}_{\mathrm{r}} \in \mathrm{R}^{\mathrm{L}_{1}}, \mathrm{~V}_{\mathrm{r}} \in \mathrm{R}^{\mathrm{L}_{2}}$ and $\mathrm{W}_{\mathrm{r}} \in \mathrm{R}^{\mathrm{L}_{3}}$ are vectors on three modes (one spectral and two spatial).Here $\mathrm{M}$ is represented as the number of rank-1 tensors used to restore whole tensor $\mathrm{O}, \lambda_{\mathrm{r}}$ is the weight value. There is no straight forward solution to $\mathrm{M}$. As the rank of a three order tensor is equivalent to the minimal number of triads necessary to describe the tensor.

\subsection{Improved K-SVD Algorithm}

The K-SVD algorithm is solved with four sub-minimization problem.

\subsubsection{Sparse Coding and Dictionary Learning}

The first minimization problem is

$\left(\alpha^{v+1}, D^{v+1}\right)=\arg \min _{\alpha, D} \mathcal{J}\left(\alpha, D, f_{v}, u_{v}, \Theta_{v}\right)$

The problem is split into two convex sub problems corresponding to the so-called sparse coding step and the dictionary learning step, respectively. Let $v 1$ be an inner iteration number, then $\alpha_{v+1}$, and $D_{v+1}$ can be obtained by solving the following two minimization problems iteratively:

$$
\begin{aligned}
& \text { Sparse Coding (Conjugated OMP) } \\
& \begin{array}{c}
\alpha^{v_{1}+1}=\arg \min _{\alpha} \mathcal{J}\left(\alpha, D, f_{v}, u_{v}, \Theta_{v}\right) \\
=\arg \min _{\alpha}\left\{\frac{\lambda}{2} \sum_{i=1}^{N}\left\|W_{i} D^{v_{1}} \alpha_{., i}-W_{i} R_{i} f^{v}\right\|_{2}^{2}+\right. \\
i=1 \text { Nuia., } i O
\end{array}
\end{aligned}
$$

Where $W_{i}$ the diagonal matrix is whose diagonal elements $\operatorname{are} R_{i \omega}$.

\section{Dictionary Learning: (Modified K-SVD)}

The original K-SVD is modified by the non-uniform weights. We denote 


$$
W=\left(R_{1 \omega} \ldots R_{N \omega}\right), X=\left(R_{1} f \ldots R_{N} f\right)
$$

Then

$D^{v_{1}+1}=\arg \min _{D,\left\|d_{k}\right\|_{2}=1}\left\{\left\|W \circ\left(D \alpha^{v_{1}+1}-X^{v}\right)\right\|_{F}^{2}\right\}$

Similar to the K-SVD learning algorithm [13] is the natural approach to minimize each atom $d_{k}$ as follows:

$d_{k}^{v_{1}+1}=\arg \min _{\left\|d_{k}\right\|_{2}=1}\left\|W \circ\left(E^{k}-d_{k} \alpha_{k, .}^{v_{1}+1}\right)\right\|_{F}^{2}$

In the above, $E^{k} \triangleq X^{v}-\sum_{l=1, l \neq k}^{K} d_{l}^{v_{1}} \alpha_{l, \text {. }}^{v_{1}+1}$. This is the weighted approximation problem. This is rectified by an iterative algorithm [22] as follows

$d_{k}^{v_{1}+1}=\arg \min _{\left\|d_{k}\right\|_{2}=1} \| W \circ\left(E^{k}-d_{k} \alpha_{k, .}^{v_{1}+1}\right)+$ $d k v 1 \alpha k, v 1+1-d k \alpha k, . F 2$

via SVD. This algorithm cannot be used for the unweighted case. Thus we solve the minimization problem was:

$d_{k}^{v_{1}+1}=\arg \min _{\left\|d_{k}\right\|_{2}=1} \| W \circ\left(E^{k}-d_{k} \alpha_{k, .}^{v_{1}+1}\right)+$ $\tau k d k \nu 1 \alpha k, \nu 1+1-\tau k d k \alpha k, . F 2$

to update the atoms, where $\tau_{k}=\left(d_{k}^{v_{1}}\right)^{T}\left(\frac{\sum_{i=1}^{N} W_{i}}{N}\right) d_{k}^{v_{1}}$. Thus the modified scheme reduces the original K-SVD algorithm when all weights are the same.

The modified K-SVD algorithm for weighted norm are as follows:

Select the index set of patches $S_{k}$ That use atom $d_{k}$

$$
S_{k}=\left\{i: \alpha_{k, i}^{v_{1+1}} \neq 0, i \leq i \leq N\right\} .
$$

Let $\tau_{k}=\left(d_{k}^{v_{1}}\right)^{T}\left(\frac{\sum_{i=1}^{N} W_{i}}{N}\right) d_{k}^{v_{1}}$, the residual is calculated for each image patch

$$
e_{i}^{\sim k}=W_{i}\left(R_{i} f^{v}-D^{v_{1}} \alpha_{., i}^{v_{1}+1}\right)+\tau_{k} d_{k}^{v_{1}} \alpha_{k, i}^{v_{1}+1}
$$

Set $\tilde{E}^{k} \in \mathbb{R}^{n_{1} n_{2} \times\left|S_{k}\right|}$ with its columns being the $e_{i}^{\sim k}$ and update $d_{k}^{v_{1}+1}$ by minimizing

$$
\left(d_{k}^{v_{1}+1}, \beta^{*}=\arg \min _{\left\|d_{k}\right\|_{2}=1, \beta}\left\|\tilde{E}^{k}-\tau_{k} d_{k} \beta^{T}\right\|_{F}^{2}\right.
$$

Where $\beta \in \mathbb{R}^{\left|S_{k}\right|}$. The rank-one approximation problem can be solved using SVD decomposition of $\tilde{E}^{k}$

Then we replace $\alpha_{k, i}^{v_{1}+1}, i \in S_{k}$ by relevant elements of $\beta^{*}$ In our experiment, the inner iteration number is chosen as $v_{1}=10$.

\subsubsection{Reconstruction}

This minimization problem is solved as follows, since $\mathcal{J}$ is quadratic with respect to $f$, thus

$$
\begin{aligned}
& \left.f^{v+1}=\left(d(\omega \circ \omega)+\lambda \sum_{i=1}^{N} R_{i}^{T} d\left(\left(R_{i} \omega\right) \circ\left(R_{i} \omega\right)\right) R_{i}\right)\right)^{-1} \\
& \quad \times\left(d(\omega \circ \omega) g+\lambda\left(\sum_{i=1}^{N} R_{i}^{T} d\left(\left(R_{i} \omega\right) \circ\left(R_{i} \omega\right)\right) R_{i}\right) \times\right. \\
& \left.D^{v+1} \alpha_{., i}^{v+1}\right)
\end{aligned}
$$

Where $d(\omega \circ \omega)$ represents $\operatorname{diag}(\omega \circ \omega)$ and $\mathrm{R}_{\mathrm{i}}$ is a diagonal matrix. So the inverse matrix can be directly obtained.

Noise Clustering (Exception Step): The solution to the minimization problem is $u^{v+1}$ and it can be computed by

$$
u_{., l}^{v+1}=\frac{\frac{r_{1}^{v}}{\sigma_{1}^{v}} \exp \left(\left[-T_{l}\right)\right.}{\sum_{s=1}^{M} \frac{r_{s}^{v}}{\sigma_{s}^{v}} \exp \left(\left[-T_{s}\right)\right.}
$$

Parameter Estimation: The minimization for this step is

$$
\begin{aligned}
\Theta^{v+1}= & \underset{\Theta, \sum r_{l}=1}{\arg \min } \mathcal{J}\left(\alpha^{\nu+1}, \mathbf{D}^{v+1}, f^{v+1}, \mathbf{u}^{v+1}, \Theta\right) \\
= & \underset{\Theta, \sum r_{l}=1}{\arg \min }\left\{\begin{array}{l}
\frac{\lambda}{2} \sum_{i=1}^{N}\left\|\mathbf{R}_{i} w \circ\left(\mathbf{D}^{v+1} \alpha_{\cdot, i}^{v+1}-\mathbf{R}_{i} f^{v+1}\right)\right\|_{2}^{2} \\
+<\mathbf{u}^{v+1}+\lambda \sum_{i=1}^{N} \mathbf{R}_{i} \mathbf{u}^{v+1}, \mathbf{1}>\ln \frac{\sigma_{l}}{r_{l}} \\
+\frac{1}{2}\left\|w \circ\left(g-f^{v+1}\right)\right\|_{2}^{2}
\end{array}\right\}
\end{aligned}
$$

From equation $\frac{\partial \mathcal{J}}{\partial \Theta}=0$, we get the closed-form solution of $\Theta^{v+1}$ :

$$
\begin{aligned}
& r_{l}^{v+1}=\frac{\left\langle u_{., l}^{v+1}, \mathbf{1}>+\lambda<\mathbf{M} u_{., l}^{v+1}, \mathbf{1}>\right.}{<\mathbf{1}, \mathbf{1}>+\lambda<\mathbf{M 1}, \mathbf{1}>} \\
& \left(\sigma_{l}^{2}\right)^{v+1}=\frac{<u_{., l}^{v+1},\left(g-f^{\nu+1}\right) \circ\left(g-f^{v+1}\right)>}{<u_{\cdot, l}^{v+1}, \mathbf{1}>+\lambda \sum_{i=1}^{N}<\mathbf{R}_{i} u_{., l}^{v+1}, \mathbf{R}_{i} \mathbf{1}>} \\
& +\frac{\lambda \sum_{i=1}^{N}<\mathbf{R}_{i} u_{., l}^{v+1},\left(\mathbf{D}^{v+1} \alpha_{,, i}^{v+1}-\mathbf{R}_{i} f^{v+1}\right) \circ\left(\mathbf{D}^{v+1} \alpha_{., i}^{v+1}-\mathbf{R}_{i} f^{v+1}\right)>}{<u_{., l}^{v+1}, \mathbf{1}>+\lambda \sum_{i=1}^{N}<\mathbf{R}_{i} u_{., l}^{v+1}, \mathbf{R}_{i} \mathbf{1}>} .
\end{aligned}
$$

\subsection{Denoising}

The signal dominant components are combined removing the noise tensors. After the noise components are removed, the noise free image is obtained by reconstructing the signal dominant components.

The tensors are reconstructed to form the noise free HSI data by the formula

$$
\widehat{S}=\sum_{\mathrm{r}=1}^{\mathrm{k}} \lambda_{\mathrm{r}} \mathrm{U}_{\mathrm{r}} \circ \mathrm{V}_{\mathrm{r}} \circ \mathrm{W}_{\mathrm{r}}
$$

The value of $\mathrm{K}$ is the number of signal dominant tensors.

\section{EXPERIMENTAL RESULTS}


The proposed algorithm is applied in 3 set of HSI data. The HIS cannot be taken as an image itself. The values are to be plotted as an image for our visualization. Thus a set of values of the received image is plotted as an image for our visualization. The values are plotted as an image for the original data and for the denoised data. The original values are not plotted fully, only certain area shown for the visualization for a clear idea of the HSI image. The three images with their denoised output is shown in figure 5-7,

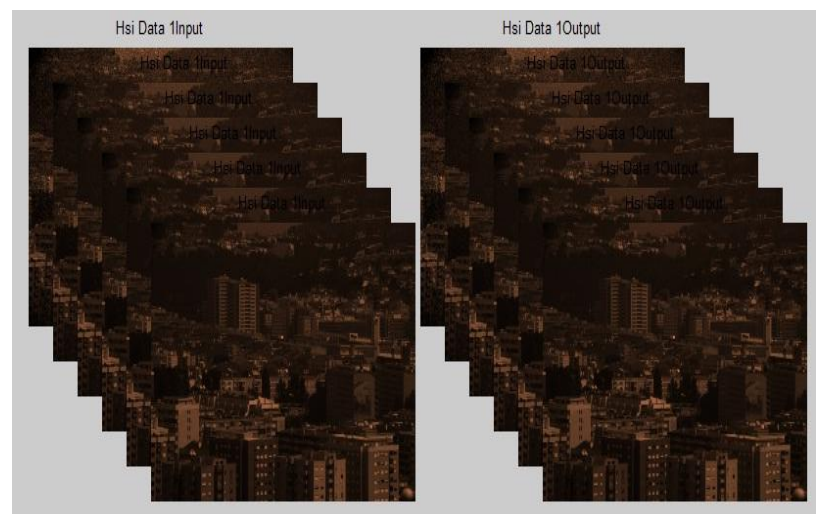

Fig- 5: HSI Data set 1 for HSI image visualization and the Denoised data set 1

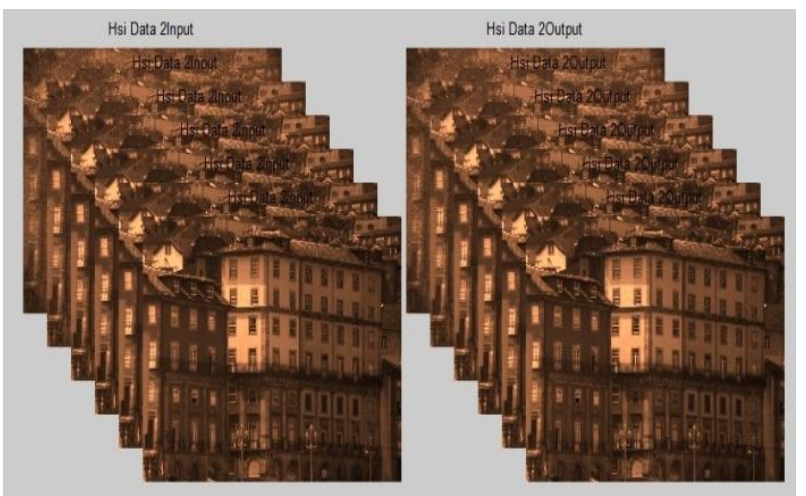

Fig-6: HSI Data set 2 for HSI image visualization and the Denoised data set 2

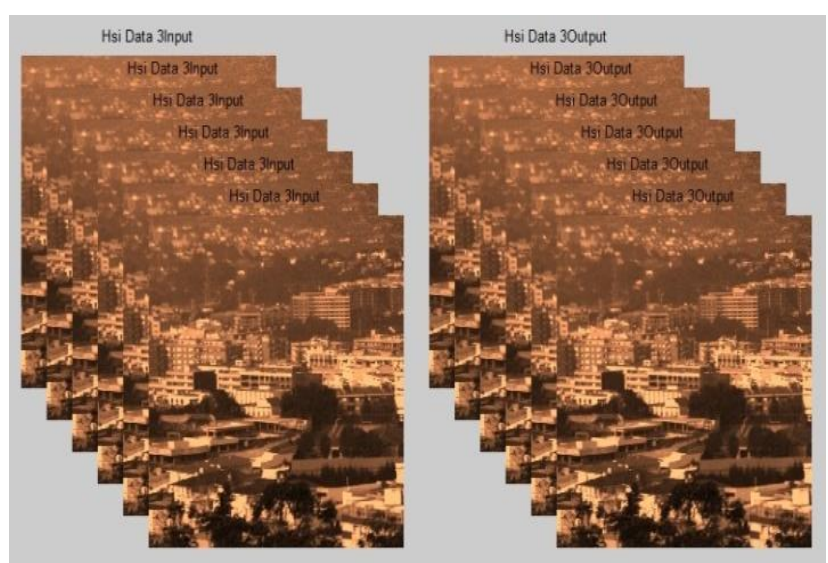

Fig-7: HSI Data set 3 for HSI image visualization and the Denoised data set 3

The effectiveness of the proposed algorithm is proved by comparing the proposed model with several competitive methods: Spectral-Spatial Adaptive Total Variation
(SSAHTV), Multidimensional Wiener Filtering (MWF) and Rank-1 Tensor Decomposition (R1TD). In the algorithm SSAHTV [15] and MWF [13] the correlation between the spatial and spectral bands is not simultaneously considered thus may lead to inter-dimensional information loss. The application of tensor product may lead to information compression and loss of spatial details. The R1TD [24] provides clear view than that of the other two but it deals with only Additive white and Gaussian noise. The proposed algorithm deals mixed noise like impulse, GaussianGaussian, Gaussian-impulse. Also it provides a higher PSNR than that of the existing system.

The PSNR is the ratio between the maximum possible power of a signal and the power of corrupting noise that affects the fidelity of its representation. The PSNR for the Existing System is compared with the proposed algorithm in the Table I. This comparison confirms that proposed method is has higher values than that of the existing systems. Also the existing system deals only with single noise whereas this deals with mixed noise.

Table-1: PSNR comparison for the existing and proposed system

\begin{tabular}{|l|l|l|l|l|}
\hline $\begin{array}{l}\text { Band } \\
\text { No. }\end{array}$ & MWF & SSAHTV & R1TD & $\begin{array}{l}\text { Improved K- } \\
\text { SVD }\end{array}$ \\
\hline 1 & 24.48 & 28.40 & 30.39 & 42.79 \\
\hline 2 & 24.66 & 26.44 & 30.43 & 40.38 \\
\hline 3 & 25.48 & 28.43 & 30.43 & 38.30 \\
\hline 4 & 24.31 & 28.21 & 30.20 & 37.39 \\
\hline 5 & 24.73 & 29.02 & 29.99 & 37.15 \\
\hline 6 & 23.73 & 27.56 & 29.52 & 37.27 \\
\hline
\end{tabular}

The graph is plotted with the PSNR values provided in the table in chart-1.

\section{PSNR Comparison}

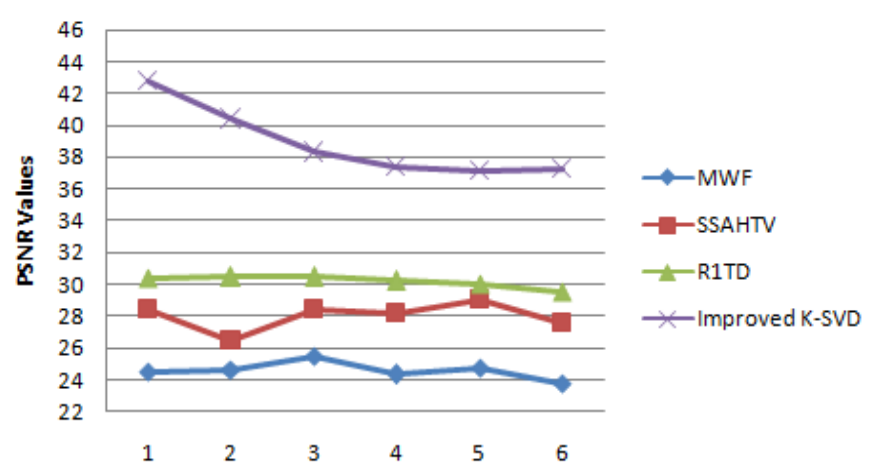

Chart-1: Graph providing the comparison of existing and the proposed system.

\section{CONCLUSIONS}

We provide a new algorithm to remove mixed noise using the PDF. By combing the sparsity regularization and dictionary learning techniques, a novel and efficient model is designed to remove mixed noise such as impulse noise, Gaussian-Gaussian mixture, and Gaussian plus impulse 
noise. Also it considers both spatial and spectral views of the hyperspectral image thus provide data quality in terms of both visual inspection and image quality.

\section{REFERENCES}

[1] Acito, N., Diani, M., Corsini, G., 2010. Hyperspectral signal subspace identification in the presence of rare signal components. IEEE Trans. Geosci. Remote Sens. 48 (4), 1940-1954.

[2] Acito, N., Diani, M., Corsini, G., 2011a. Signaldependent noise modeling and model parameter estimation in hyperspectral images. IEEE Trans. Geosci. Remote Sens. 49 (8), 2957-2971.

[3] Acito, N., Diani, M., Corsini, G., 2011b. Subspacebased striping noise reduction in hyperspectral images. IEEE Trans. Geosci. Remote Sens. 49 (4), 1325-1342. Akaike, H., 1974.

[4] Ana Rovi., 2010. Analysis of $2 \times 2 \times 2$ Tensors. master's thesis

[5] M. Elad and M. Aharon, "Image denoising via learned dictionaries and sparse representation," in Proc. IEEE Comput. Vis. Pattern Recognit.,Jun. 2006, pp. 895-900.

[6] M. Elad and M. Aharon, "Image denoising via sparse and redundant representations over learned dictionaries," IEEE Trans. Image Process.,vol. 15, no. 12, pp. 3736-3745, Dec. 2006.

[7] M. Aharon, M. Elad, and A. Bruckstein, "The KSVD: An algorithm for designing of overcomplete dictionaries for sparse representations, 'IEEE Trans. Image Process., vol. 54, no. 11, pp. 4311-4322, Nov. 2006.

[8] J. Mairal, M. Elad, and G. Sapiro, "Sparse representation for color image restoration," IEEE Trans. Image Process., vol. 17, no. 1, pp. 53-69, Jan. 2008.

[9] Bourennane, S., Fossati, C., Cailly, A., 2011. Improvement of target-detection algorithms based on adaptive three-dimensional filtering. IEEE Trans. Geosci. Remote Sens. 49 (4), 1383-1395.

[10] Brett W. Bader and Tamara G. Kolda MATLAB Tensor Classes for Fast Algorithm Prototyping

[11] Bro, R., Kiers, H.A.L., 2003. A new efficient method for determining the number of components in PARAFAC models. J. Chemometr. 17 (5), 274286Karami, A., Yazdi, M., Asli, A.Z., 2011. Noise reduction of hyperspectral images using kernel nonnegative tucker decomposition. IEEE J. Sel. Top. Signal Process. 5 (3), 487-493.

[12] Landgrebe, D., 2002. Hyperspectral image data analysis. IEEE Signal Process. Mag. 19 (1), 17-28.

[13] Letexier, D., Bourennane, S., 2008. Noise removal from hyperspectral images by multidimensional filtering. IEEE Trans. Geosci. Remote Sens. 46 (7), 2061-2069.

[14] Othman, H., Qian, S.-E., 2006. Noise reduction of hyperspectral imagery using hybrid spatial-spectral derivative-domain wavelet shrinkage. IEEE Trans. Geosci. Remote Sens. 44 (2), 397-408.
[15] Yuan, Q., Zhang, L., Shen, H., 2012. Hyperspectral image denoising employing a spectral-spatial adaptive total variation model. IEEE Trans. Geosci. Remote Sens. 50 (10), 3660-3677.

[16] H. Wang and R. Haddad, "Adaptive median filters: New algorithms and results," IEEE Trans. Image Process., vol. 4, no. 4, pp. 499-502, Apr.1995.

[17] Y. Xiao, T. Zeng, J. Yu, and M. K. Ng, "Restoration of images corrupted by mixed Gaussian-impulse noise via 11-10 minimization,"Pattern Recognit., vol. 44, no. 8, pp. 1708-1720, Aug. 2011.

[18] E. Lopez-Rubio, "Restoration of images corrupted by Gaussian and uniform impulsive noise," Pattern Recognit., vol. 43, no. 5,pp. 1835-1846, 2010.

[19] J. Liu, H. Huang, Z. Huan, and H. Zhang, "Adaptive variational method for restoring color images with high density impulse noise," Int. J.Comput. Vis., vol. 90, no. 2, pp. 131-149, 2010

[20] J. Bilmes. (1997). A Gentle Tutorial On The Em Algorithm And Its Application To Parameter Estimation For Gaussian Mixture And Hidden Markov Models Available: Http://Citeseerx.Ist.Psu.Edu/Viewdoc/Summary?Doi $=10.1 .1 .28 .613$

[21] N. Srebro And T. Jaakkola, "Weighted Low-Rank Approximations," In Proc. 20th Int. Conf. Mach. Learn., 2003, Pp. 720-727.

[22] S. Ko And Y. Lee, "Center Weighted Median Filters And Their Applications To Image Enhancement," Ieee Trans. Circuits Syst., Vol. 38, No. 9, Pp.984993, Sep. 1991.

[23] Xian Guo A, Xin Huang A, $\Uparrow$, Liangpei Zhang A, Lefei Zhang"Hyperspectral Image Noise Reduction Based On Rank-1 Tensor Decomposition", Isprs Journal Of Photogrammetry And Remote Sensing 83 (2013) 50-63

\section{BIOGRAPHIES}

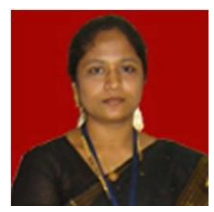

Prof.S.ShajunNisha has completed M.Phil.(Computer science) and M.Tech (Computer and Information Technology) in Manonmaniam Sundaranar University, Tirunelveli. She is a member of ISTE and IEANG and her specialization is Image Mining.

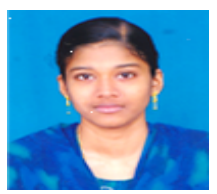

P. Thazneem Fazila has finished her B.Tech (IT) and doing her M.E.(computer Science and Engineering) in National college of engineering. She has attended so many national and international seminars, conferences and presented research papers.

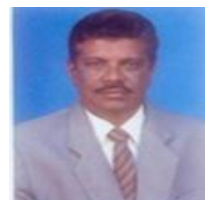

Dr. S. Kother Mohideen has been working as a Professor in the Department of Computer Science and Engineering, National College of Engineering, Tirunelveli. He is also Research convenor of R\&D Department. He is obtained M.Tech degree and $\mathrm{Ph} . \mathrm{D}$ degree from Manonmaniam Sundraranar University, Tiruneveli. He is the member of IEEE and IEANG. 